\title{
Comparison of different configuration space representations for path planning under combinatorial method
}

\author{
Sanjoy Kumar Debnath, Rosli Omar, Nor Badariyah Abdul Latip \\ Faculty of Electrical and Electronic Engineering, Universiti Tun Hussein Onn Malaysia, Malaysia
}

\begin{tabular}{l}
\hline \hline Article Info \\
\hline Article history: \\
Received Dec 15, 2018 \\
Revised Feb 16, 2019 \\
Accepted Feb 27, 2019 \\
\hline
\end{tabular}

\section{Keywords:}

Cell-decomposition

Path planning

Potential field

Visibility graph

Voronoi diagram

\begin{abstract}
The use of autonomous vehicle/robot has been adopted widely to replace human beings in performing dangerous missions in adverse environments. Keeping this in mind, path planning ensures that the autonomous vehicle must safely arrive to its destination with required criteria like lower computation time, shortest travelled path and completeness. There are few kinds of path planning strategies, such as combinatorial method, sampling based method and bio-inspired method. Among them, combinatorial method can accomplish couple of criteria without further adjustment in conventional algorithm. Configuration space provides detailed information about the position of all points in the system and it is the space for all configurations. Therefore, C-space denotes the actual free space zone for the movement of robot and guarantees that the vehicle or robot must not collide with the obstacle. This paper analyses different C-Space representation techniques under combinatorial method based on the past researches and their findings with different criteria such as optimality, completeness, safety, memory uses, real time and computational time etc. Visibility Graph has optimality which is a unique from other.
\end{abstract}

Copyright $\odot 2019$ Institute of Advanced Engineering and Science. All rights reserved.

\section{Corresponding Author:}

Rosli Omar,

Faculty of Electrical \& Electronic Engineering,

Universiti Tun Hussein Onn Malaysia,

Parit Raja, Batu Pahat-86400, Johor, Malaysia.

Email: roslio@uthm.edu.my

\section{INTRODUCTION}

Path planning algorithm is important to produce an optimal path that enables the shortest distance movement of a vehicle or robot from a starting point to a target point with minimum computational time. The path planning algorithm should also hold complete criterion so that it is able to find a path, if one exists. Besides that, the vehicle's safety, memory usage for computing and the real-time applicable algorithm are also significant [1-6]. Path planning approaches, in general, can be classified in three ways, such as combinatorial method, sampling-based method, and bio-inspired method. The combinatorial path planning creates a route by resolving queries along the way. Figure 1 illustrates the classification of path planning approaches.

It comprises mainly two methods like C-space representation technique and graph search algorithm. This paper focuses on C-space representation techniques. Road map (RM), cell decomposition (CD) and potential field $(\mathrm{PF})$ techniques are among the ways of space representation. Road map technique is also divided into two categories - visibility graph (VD) and Voronoi diagram (VD) [7].

Visibility graph (VG) algorithm finds a path by connecting all the visible vertices of an obstacle including a starting and an ending point. After that, a shortest path is searched from the connected graph. In cell decomposition (CD) method, the first step is to decompose the configuration space into cells and then it finds a cell which is not occupied by any obstacle. The Voronoi diagram (VD) is an equidistant point from 
two or all the nearest obstacles and by connecting all these points, a path can be found [6], [8] and [9]. $\mathrm{PF}$ algorithm is a path planning technique based on attractive and repulsive potential [9-10].

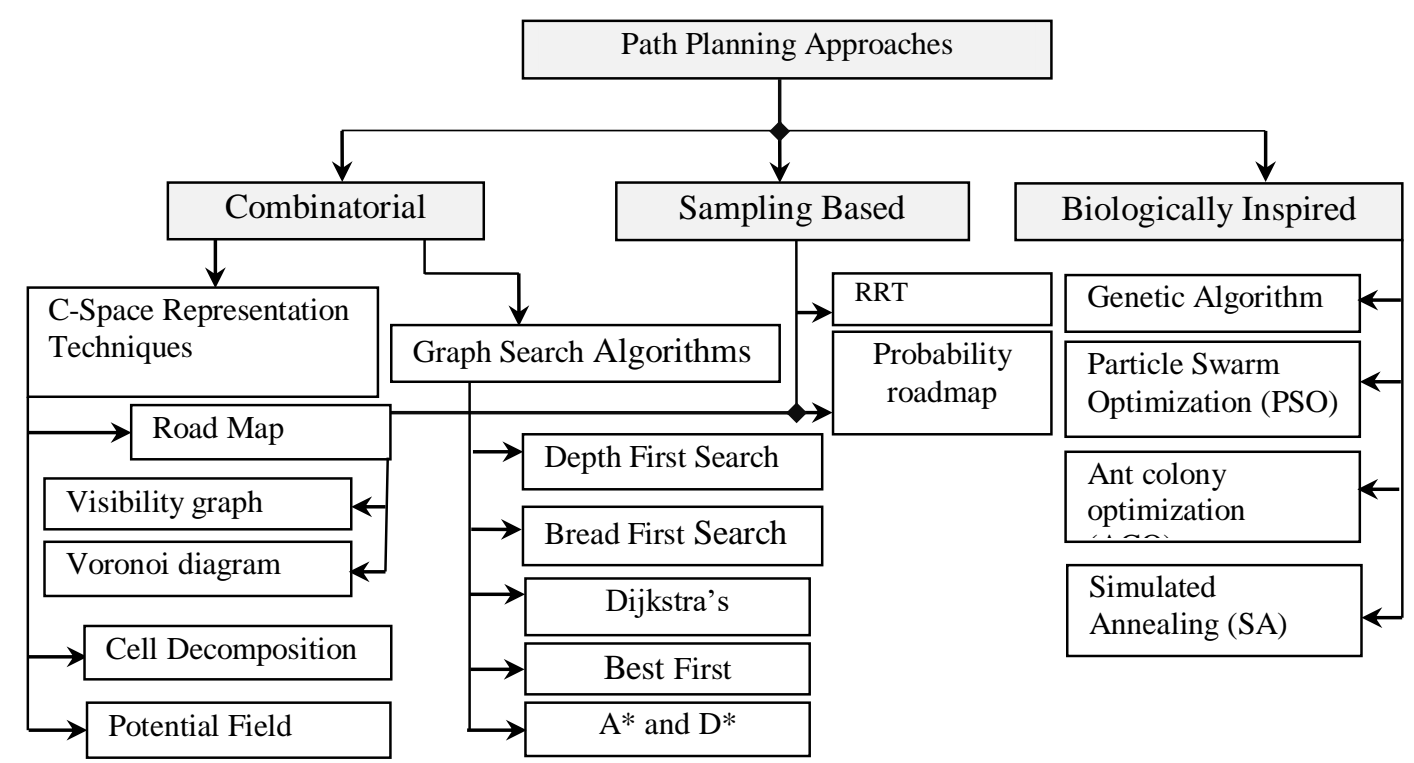

Figure 1. Classification of path planning approach

Besides the combinatorial method, rapidly exploring random tree (RRT) is an example of samplingbased path planning where pre-registration of the design space is not required. Here firstly, the starting and the target points are defined. Then, it considers the starting point as the foundation for the tree, based on which a new branch is grown until it reaches the target point [11-13]. Probabilistic roadmap (PRM) technique is a path-planning algorithm that takes arbitrary samples from the configuration space by checking the accessible free space and dodging the crashes to find a way. A local planner is used to join these configurations with close-by configurations. A graph search algorithm is applied after adding the initial and goal configurations to determine a path [14-15].

The bio-inspired methods are the nature-motivated algorithms. Various examples of bio-inspired methods are the Genetic algorithm (GA), Particle Swarm Optimization (PSO), Simulated annealing (SA), and Ant Colony Optimization (ACO). The natural selection process to find a path stimulates GA. There are two steps of this path planning. Firstly, it sets up a C-space in a free space and then, it finds a path from the start point to target point [16]. PSO is an established meta-heuristic populace based calculation, initially presented by Kenney and Eberhart in 1995, to resolve the global optimization issues in the light of shared conduct of natural populaces [17]. A PSO based path-planning algorithm tries to solve the issues of multitarget path planning models regarding robot's energy consumption and path's safety [18]. The ACO emulates an ant to make a path when the food source is confirmed. The ant separates its route to the food source with pheromones for tracking purpose. In ACO, the path in between the initial and target points are haphazardly generated [19-21]. SA algorithm looks like the warming and cooling process of metals to regulate the inner structure of its properties. Higher temperature procedure investigates a bigger region of search space. The gradual probabilistic properties permit this algorithm to avoid local minima and maxima solutions [10-12], [16], [21-24].

C-space representation technique used in VG, VD, CD and PF are studied because it is used in combinatorial path planning. C-space provides detailed information about the position of all points in the system and it is the space for all configurations. An illustration of a $\mathrm{C}$-space for a circular vehicle is shown in Figure 2.

It assumes the vehicle or robot as a point and adds the area of the obstacles so that the path planning can be done more efficiently. $\mathrm{C}$-space is obtained by adding the vehicle radius while sliding it along the edge of the obstacles and the border of the search space.

In Figure 2(a), the obstacle-free area is represented by the white cells. The vehicle is shown by a dark black dot hovered with dim shading. Three possible collision-free paths are drawn and those are dotted, semi-dabbed and solid lines to achieve the target/goal configuration from start/initial configuration 
considering that $\mathrm{C}$-space is not created. Conversely, when the workspace is considered as C-space, as appeared in Figure 2(b), the robot has just a single possible way. This also reveals that the free space has been reduced while the obstacles' region has been enlarged. Therefore, C-space denotes the actual free space zone for the movement of robot and it guarantees that the vehicle or robot must not collide with the obstacle.

In this case, the first step is to construct the $\mathrm{C}$-space environment and the second step is to generate a path using a graph search algorithm. Dijkstra's, A* or D*, Depth-first search, Bread First, Best first are the algorithms to find the optimal path depending on the generated path [1], [25-26].

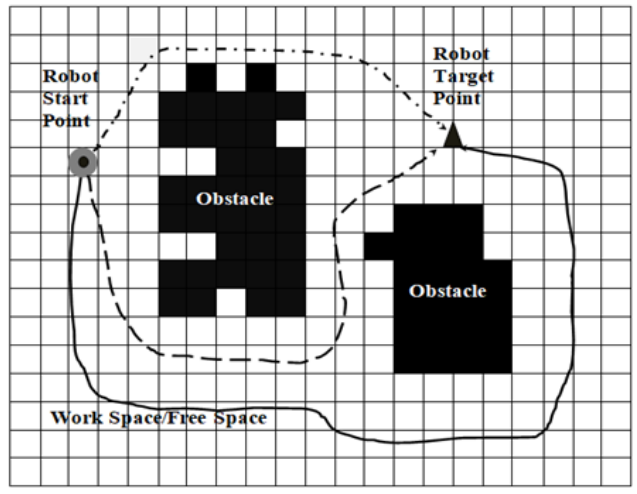

(a)

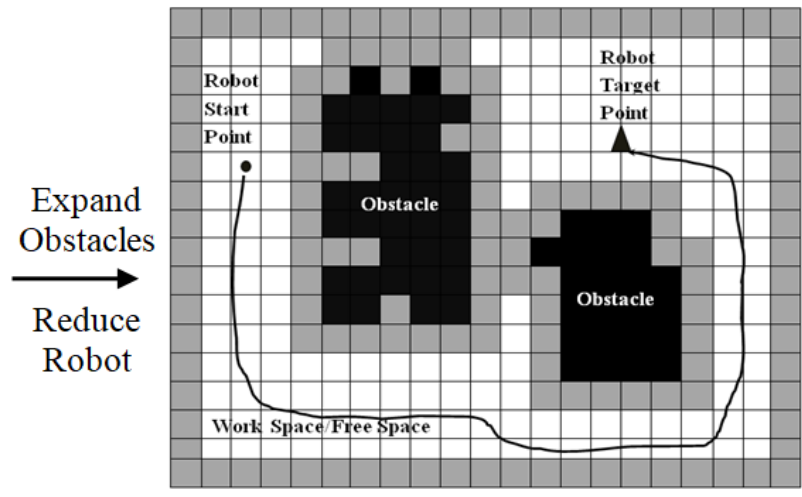

(b)

Figure 2. Configuration space for path planning

\section{COMBINATORIAL PATH PLANNING}

\subsection{Visibility Graph (VG)}

VG is a path planning method based on combinatorial system [4] that finds out a path by solving queries along the way. VG is used in many applications such as graphics and robotics [27]. It is a set of polygonal configuration in a plane (either two or three dimensional) at an undirected graph where vertices are the obstacles' vertices and the edges are the pairs of vertices. An open line segment between two vertices does not intersect any obstacle [28-29]. In VG, the vertices include starting point and target point [28]. It is important that obstacles are in an open set to ensure that the shortest path exists. To proceed with the visibility graph in search space, the sets of vertices that are mutually visible need to be discovered. This implies that for each pair of vertices, it needs to be tested whether the connecting line segment hits any obstacle [30]. The connected vertices with canter shown in Figure 3.

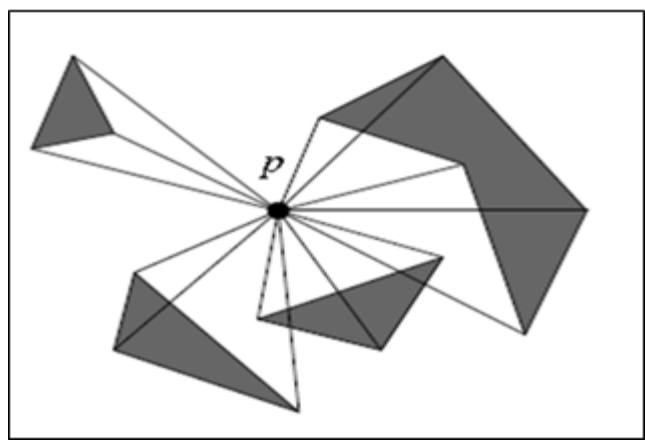

Figure 3. The connected vertices with canter $\mathrm{p}[30]$

Extensive investigations have been done on VG to reduce the computational time. For instance, researchers applied VG in [5] based on the polygon aggregation. The main idea of this technique is to cluster small obstacles and merge the polygons after clustering. Further, in [14], an algorithm was proposed to separate the C-spaces in many parts and VG was utilized to assemble each part at parallel direction. The shortest path was determined by integrating each partial minimum distance path. Besides that, a study in 
[31] enhanced the VG algorithm by sharing local information between multiple robots to overcome its disadvantages. The proposed algorithm generated polygons from a series of linked segments and merged them, if necessary. The result of the sharing information was compared with the non-sharing information by VG and the proposed algorithm was more effective. The modified VG in developed a Virtual rubber band visibility graph (VRBVG) method to generate a VG under the assumption that C-spaces were unknown and located outside the vehicle of sonar coverage. They used torpedo-type under actuated vehicles to travel in an unknown underwater condition.

\subsection{Voronoi Diagram (VD)}

The aim of VD is to find a path far from the obstacles [24]. The idea behind the VD is to generate a line segment called Voronoi Edge which is equidistant from all the points of the obstacle area in C-space [6], [33]. The point, where the Voronoi Edge joins with each other, is called Voronoi Vertex. An example of VD representation that is used for path planning is displayed in Figure 4. The resulting path is shown in solid black line. As per the illustration of Figure 4, VD has edges to give a maximum clearance path among set of obstacles in the C-space. If a vehicle traverses the planned path, it is guaranteed that the vehicle must not intersect any obstacle. However, the VD generated paths are not optimal in terms of length.

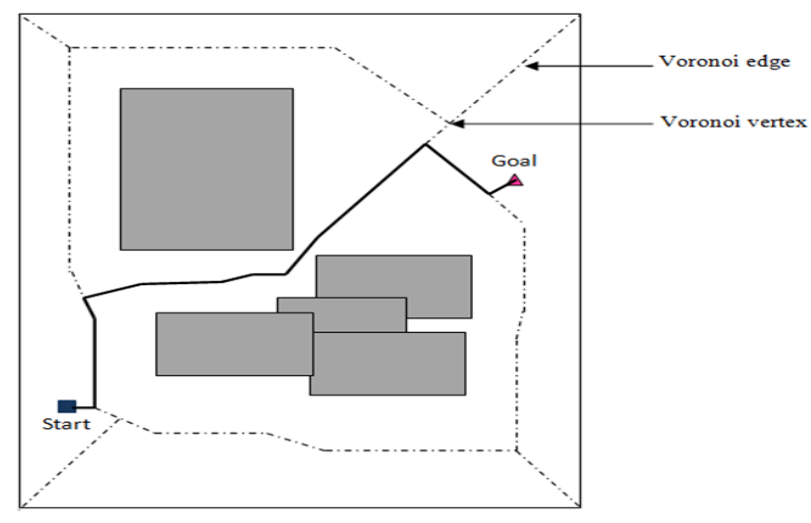

Figure 4. The dashed lines in Voronoi diagram are the set of points equidistant to obstacles. The path is shown in solid darker lines.

In [6], improvements were done on VD to follow the kinematic constraint of an aircraft in three steps. Firstly, an initial diagram was generated by the fundamental VD. Secondly, the initial diagram was enhanced by smoothing the impractical corner of all paths from starting point to target point. Then, the cost of the edge of improved VD was modeled and weighted. Finally, the optimal path was selected using the Dijkstra's algorithm from the smooth path. Improved VD was much lower compared to the fundamental one. Another enhancement was done on the fundamental VD in [24] with Delaunay triangulation. The algorithm was tested with 25 different settings. The outcomes revealed that the improved VD was computationally less expensive and it responded in shorter time. But it produced a path that might not be the shortest one and this was the drawback of this algorithm. The modified VD was applied in on unmanned air vehicle in a dynamic environment. Here, the path was created by using the radar threat field based VD. Then Dijkstra's algorithm was applied to calculate the shortest distance. To reduce the computational time, in [33] the images were captured and then clustered into a smaller group. A smooth path for a robot was also created from a path planning algorithm based on the fuzzy interference mechanism.

\subsection{Cell-Decomposition (CD)}

CD method mainly finds an obstacle-free cell and builds a finite graph for these cells [11]. It breaks the environment into cells and ensures that each cell is discrete, non-overlapping and not occupied by any obstacle. A finite graph is built by relegating every cell as a hub. In cell decomposition method, the first step is to decompose the configuration space into cells. After that, the connectivity graph is built. Each node of the generated graph represents a cell and they are between two nodes represent two corresponding cells adjoining each other. Then the connectivity graph from initial to end point is determined [9]. There are several types of cell decomposition methods such as regular grid decomposition (RG), adaptive cell decomposition (ACD) and exact cell decomposition (ECD) [9], [29]. 
In order to overcome the drawback of CD method, an improvement was made in [11] to increase the efficiency of the algorithm by dividing the cell quarterly. Then, the cell was checked to find out the presence of any obstacle. After that, the cell was divided again in quarter. This method was used to achieve the optimum path. Exact cell decomposition method and exhaustive path planning were used in [35]. The step for this algorithm began with the mineable area that was divided into an exact cell. Then, each cell coverage path was generated with covering direction and the generated graph paths were based on the adjacency graph. By considering all graph paths with all covering directions, a moving path was generated. Lastly, a shortest moving path for all graph paths was determined and the exhaustive path was generated. The non-optimal path is one of the drawbacks of CD. A research in [36] had derived three formulas using L1 norm, squared L2, and $\mathrm{L} \infty$ norm. As an alternative to use the cell's midpoints in the fundamental CD, this technique used three formulas to define the metrics. A study in [37] generated CD directly into workspaces. They applied the path planning for palletizing and common handling jobs. The algorithm produced cylindrical cell decomposition in the workspace of a six DOF robot to speed up the time without any requirement of an obstacle's transformation in a workspace into configuration spaces

\subsection{Potential Field (PF)}

$\mathrm{PF}$ was firstly suggested by Khatib [38]. This path planning algorithm is based on the attractive potential and repulsive potential in the configuration space consisting of a starting point, a target point, and obstacles. The vehicle is represented as a point that moves under the potential field. The target point acts as an attractive potential while the obstacles in configuration spaces simulate repulsive potential. Repulsive potential tasks in path planning are to avert the vehicles that may collide with any existing obstacle in configuration spaces, moving under the influences of attractive forces [9-10].

A global off-line path planning methodology was applied using an energy-based approach recognized as Artificial Potential Field (APF) for Multi-Robot Systems (MRSs). Based on the potential field, a developed artificial potential field (APF) path planning technique was hosted and it was more operative in finding the shortest path [39]. Another potential field method used the kinematics of a six-wheel rover for motion on rough 3D terrain where comparative significance of the paths was obtained from four dissimilar cost functions with respect to energy, traction force, slip and deviation from a straight line. Wide experiments and simulations revealed that this technique was better in obtaining paths [40].

\section{DISCUSSION ON COMBINATORIAL PATH PLANNING}

Few combinatorial path planning methods are tabulated in Table 1 and 2 exhibits their pros and cons. It reveals that VG is capable to resolve path planning issue by finding the shortest distance without the probability of local minima [4], [7], [31], [41] and [45]. It satisfies two criteria for optimal path planning in terms of completeness and minimum travelled path [4], [5], [7] and [46]. Besides that, it is simple to implement [31]. The limitation of VG is the computational time since it increases with the increasing quantity of obstacles in C-spaces.

The main advantage of VD is the minimum computational time and the ability to attain the global optimal solution [18]. Furthermore, it is a complete algorithm since it is capable to find a way without the chance of local minima occurrence. In any case, the weakness of VD is that it is unable to create the shortest path [7] for which there is a high possibility of wastage of energy consumption and cost.

The advantages of $\mathrm{CD}$ are that it gives guarantee to find a collision-free path, if exists and is manageable by a robot. Hence, it is a complete algorithm since the robot can explore the path without the risk of local minima occurrence [8]. Nevertheless, the problem of CD is that if the produced cell is too rough, then it will not be possible to accomplish the minimum path length. On the other hand, if the cell is too small, then longer computation time is needed [1], [33], and [42]. The cell decomposition strategy also does not work extremely well in a dynamic situation and in real-time condition [8], [11], and [33]. It is necessary for $\mathrm{CD}$ to adjust with the condition as required; e.g., in exact $\mathrm{CD}$, the cells are not predefined, but they are nominated based on the location and shape of the obstacles within the C-space [32].

One major shortcoming of PF method is local minima at which the vehicle can get stuck. As PF method leads the vehicle in the direction of a minima within the field, it is not assured that the minima is the global one. This makes the PF recognized as a local method because the outcome of the field is almost exclusively based on the nearby obstacles of the vehicle. Distant obstacles may have small or even no effect on the vehicle's motion. Additional drawback of PF method is its least satisfactory avoidance of obstacle when the vehicle maneuvers through close-fitting environment [34]. This is because the PF is normally modeled as continuous and differentiable occupation, leading to a vague description of the obstacle's shape and dimension. 
Table 1. Comparison of Different C-Space Representations under Combinatorial Method

\begin{tabular}{cccccccc}
\hline \multicolumn{2}{c}{ Method } & Optimal Path & Computational Time & Real time & Memory & Safety & Completeness \\
\hline \multirow{2}{*}{ RM } & VG & $\sqrt{2}$ & $\times$ & $\times$ & $\times$ & $\times$ & $\sqrt{ }$ \\
& VD & $\times$ & $\sqrt{ }$ & $\times$ & $\sqrt{ }$ & $\times$ & $\sqrt{ }$ \\
& RG & $\times$ & $\sqrt{ }$ & $\times$ & $\times$ & $\sqrt{ }$ & $\times$ \\
CD & ACD & $\times$ & $\sqrt{ }$ & $\times$ & $\sqrt{ }$ & $\sqrt{ }$ \\
& ECD & $\times$ & $\times$ & $\times$ & $\sqrt{ }$ & $\times$ \\
PF & PF & $\times$ & $\sqrt{ }$ & $\sqrt{ }$ & $\sqrt{ }$ & $\times$ \\
\hline
\end{tabular}

Table 2. Comparison of Complexity of each C-Space Representation

\begin{tabular}{ccc}
\hline \multicolumn{2}{c}{ Method } & Computational Complexity \\
\hline \multirow{2}{*}{ RM } & VG & $\mathrm{O}\left(\mathrm{n}^{2}\right)$ \\
& VD & $\mathrm{O}(\mathrm{n} \log \mathrm{n})$ \\
& RG & $\mathrm{O}(\mathrm{n} \log \mathrm{n})$ \\
$\mathrm{CD}$ & ACD & $\mathrm{O}(\mathrm{n} \log \mathrm{n})$ \\
& ECD & $\mathrm{O}(\mathrm{n} \log \mathrm{n})$ \\
$\mathrm{PF}$ & PF & --- \\
\hline
\end{tabular}

\section{FUTURE WORKS ON C-SPACE REPRESENTATION FOR PATH PLANNING}

In the future, autonomous systems will be one of the requirements in enhancing human quality of life. By applying autonomous systems, human movement from point A to point B will be faster and more efficient. In these regard Industry 4.0 standard is adopted by companies based on four design principles such as "interoperability", "information transparency", "Technical assistance", and "decentralized decisions" [43] in identifying and implementing different scenarios.

UAVs are increasingly being used to replace humans in performing risky missions at adverse environments and this meets the criterea of "technical assistance". The UAV has to traverse a pre-planned path autonomously from a starting point to the final destination and this can be described as the "decentralized decisions" that is a vital enabler to develop an autonomous industry. The Project in advance lavel can also be involved with"Interoperability" that enbles the communication with the UAV via the Internet of Things (IoT). This confirms the "Information transparency". Furthermore, the required key technologies for Industry 4.0 transformation such as artificial intelligence, IoT, machine learning, cloud systems, cybersecurity and adaptive robotics partly or fully can be implemented with the chosen algorithm to achieve the desired outcome [44].

\section{CONCLUSION}

Researchers already developed a number of path planning algorithms and their findings are compared in this paper for all the combinatorial techniques considering the nature of motion along with their advantages and drawbacks. Except that, several modifications that were proposed by different researchers to overcome the drawback of each technique are discussed in Section 2. Currently, path planning approach is multi-dimensional. To be an efficient path planning algorithm, it (i) can find an optimal collision-free path, (ii) is complete, (iii) has minimal computation time, and (iv) produces energy efficient paths. Based on the objective of the vehicle's mission and considering the outcome of the available path planning algorithms, such as computation time, completeness and safety, these algorithms can be optimized to obtain the energy efficient path planning. Each path planning technique has its own advantages and drawbacks; its application completely depends on the mission and its predefined requirements. For example, VD technique is suitable for aircraft because of its huge physical body and thus, it needs more spaces. VG performs well in less complex environment and CD is suitable for an amenable environment.

\section{ACKNOWLEDGEMENTS} Vot H131.

This research supported by Universiti Tun Hussein Onn Malaysia (UTHM) and funded by TIER-1

\section{REFERENCES}

[1] Omar, Rosli bin".Path planning for unmanned aerial vehicles using visibility line-based methods".PhD diss.,University of Leicester, 2012. 
[2] Latombe, Jean-Claude. "Robot motion planning". Springer Science \& Business Media, Vol. 124, 2012.

[3] Ganeshmurthy, M. S., and G. R. Suresh. "Path planning algorithm for autonomous mobile robot in dynamic environment." Signal Processing, Communication and Networking (ICSCN), 2015 3rd International Conference on. IEEE, 2015.

[4] T. Nguyet, N. Duy-Tung, V. Duc-Lung, and T. Nguyen-Vu. "Global Path Planning for Autonomous Robots using Modified Visibilitygraph". IEEE, 2013; vol. 13, pp. 317-321.

[5] Nguyet, Tran Thi Nhu, Tran Van Hoai, and Nguyen Anh Thi. "Some advanced techniques in reducing time for path planning based on visibility graph." Knowledge and Systems Engineering (KSE), 2011 Third International Conference on. IEEE, 2011.

[6] Chen, Peng, et al. "Research of path planning method based on the improved Voronoi diagram." Control and Decision Conference (CCDC), 2013 25th Chinese. IEEE, 2013.

[7] Omar, Rosli, and Da-Wei Gu. "Visibility line based methods for UAV path planning." ICCAS-SICE, 2009. IEEE, 2009.

[8] Glavaški, Dušan, Mario Volf, and Mirjana Bonkovic. "Robot motion planning using exact cell decomposition and potential field methods." Proceedings of the 9th WSEAS international conference on Simulation, modelling and optimization. World Scientific and Engineering Academy and Society (WSEAS), 2009.

[9] Siciliano, Bruno, Lorenzo Sciavicco, Luigi Villani, and Giuseppe Oriolo. "Robotics: modelling, planning and control." Springer Science \& Business Media, 2010.

[10] $\mathrm{Li}$, Guanghui, et al. "An efficient improved artificial potential field based regression search method for robot path planning." Mechatronics and Automation (ICMA), 2012 International Conference on. IEEE, 2012.

[11] A. Abbadi and R. Matousek.Path Planning Implementation Using MATLAB in Technical Computing Bratislava, 2014, pp. 1-5.

[12] Adiyatov, Olzhas, and Huseyin Atakan Varol. "Rapidly-exploring random tree based memory efficient motion planning." Mechatronics and Automation (ICMA), 2013 IEEE International Conference on. IEEE, 2013.

[13] Devaurs D, Siméon T, Cortés J. "Parallelizing RRT on distributed-memory architectures." InRobotics and automation (ICRA), 2011 IEEE International Conference on 2011 May 9. pp. 2261-2266.

[14] Latombe JC. "Motion planning: A journey of robots, molecules, digital actors, and other artifacts." The International Journal of Robotics Research. 1999 Nov;18(11):1119-28.

[15] Marble JD, Bekris KE."Asymptotically near-optimal planning with probabilistic roadmap spanners." IEEE Transactions on Robotics, vol. 29(2), pp.432-44, Apr 2013.

[16] Achour N, Chaalal M."Mobile robots path planning using genetic algorithms." InThe seventh international conference on autonomic and autonomous systems, Baker(ICAS 2011).2011; pp. 111-115.

[17] Eberhart R, Kennedy J. "A new optimizer using particle swarm theory." In Micro Machine and Human Science (MHS'95). Proceedings of the Sixth International Symposium on 1995 Oct 4. IEEE. 1995;pp. 39-43.

[18] Davoodi M, Panahi F, Mohades A, Hashemi SN. "Clear and smooth path planning”. Applied Soft Computing. 2015 Jul 1;32:pp.568-79.

[19] Shaogang Z, Ming L. "Path planning of inspection robot based on ant colony optimization algorithm.” In Electrical and Control Engineering (ICECE).2010 International Conference on 2010 Jun 25;IEEE. pp. 1474-1477.

[20] W. Hao and X. Xu."Immune ant colony optimization network algorithm for multi-robot path planning Proc." IEEE Int. Conf. Softw. Eng. Serv.Sci.ICSESS.2014;pp.1118-1121.

[21] Hsu, Chen-Chien, Wei-Yen Wang, Yi-Hsing Chien, Ru-Yu Hou, and Chin-Wang Tao. "FPGA implementation of improved ant colony optimization algorithm for path planning." In Evolutionary Computation (CEC), 2016 IEEE Congress on, pp. 4516-4521. IEEE, 2016.

[22] Goyal, Jitin Kumar, and K. S. Nagla. "A new approach of path planning for mobile robots." In Advances in Computing, Communications and Informatics (ICACCI, 2014 International Conference on, pp. 863-867. IEEE, 2014.

[23] Yongnian Z, Lifang Z, Yongping L. “An improved genetic algorithm for mobile robotic path planning." In Control and Decision Conference (CCDC) China 2012 May 23;IEEE. pp. 3255-3260.

[24] Gomez EJ, Santa FM, Sarmiento FH. "A comparative study of geometric path planning methods for a mobile robot: Potential field and Voronoi diagrams." In Engineering Mechatronics and Automation (CIIMA), 2013 II International Congress of 2013 Oct 23;IEEE. pp. 1-6.

[25] S. M. Lavalle, "Planning algorithm." Cambridge University, 2006.

[26] Kunigahalli, Raghavan, and Jeffrey S. Russell. "Visibility graph approach to detailed path planning in cnc concrete placement." In Automation and robotics in construction XI, pp. 141-147. 1994.

[27] Dehghani, G. and Morady, H. "An algorithm for visibility graph recognition on planar graphs." In Future Computer and Communication,ICFCC 2009. International Conference on IEEE 2009, pp. 518-521.

[28] Kaluđer, Hrvoje, Mišel Brezak, and Ivan Petrović. "A visibility graph based method for path planning in dynamic environments." In MIPRO, 2011 Proceedings of the 34th International Convention, pp. 717-721. IEEE, 2011.

[29] H. Choset et al."Principles of Robot Motion Theory, Algoriyhm and implementation.” The MIT Press, 2005.

[30] M. De berg, O. Cheong, M. Van krevald, and M. Overmars, Visibility Graphs.Springer.

[31] M. Yingchong, Z. Gang, and P. Wilfrid."Cooperative path planning for mobile robots based on visibility graph. "In Control Conference (CCC), China, 2013, pp. 4915-4920.

[32] Giesbrecht, J. "Global path planning for unmanned ground vehicles. No. DRDC-TM-2004-272." Defence Reserch And Development Suffield Alberta, 2004. 
[33] Gonzalez, Ramon, Cristian Mahulea, and Marius Kloetzer. "A Matlab-based interactive simulator for mobile robotics." In Automation Science and Engineering (CASE), 2015 IEEE International Conference on, IEEE, 2015, pp. 310-315.

[34] D. Chen, L. Zhan, X. Chen.'Mobile robot path planning based on behaviour information potential field in unknown environments." In Proceedings of the IEEE International Conference on Robotics and Biomimetics, 2004, pp. 683-687.

[35] Kim, Dae Hwan, Giang Hoang, Min-Ji Bae, Jin Wook Kim, Suk Min Yoon, Tae-Kyeong Yeo, Hong Sup, and Sang-Bong Kim. "Path tracking control coverage of a mining robot based on exhaustive path planning with exact cell decomposition." In Control, Automation and Systems (ICCAS), 2014 14th International Conference on, IEEE, 2014, pp. 730-735.

[36] Kloetzer, Marius, Cristian Mahulea, and Ramon Gonzalez. "Optimizing cell decomposition path planning for mobile robots using different metrics." In System Theory, Control and Computing (ICSTCC), 2015 19th International Conference on. IEEE, 2015, pp. 565-570.

[37] Scheurer, Christian, and Uwe E. Zimmermann. "Path planning method for palletizing tasks using workspace cell decomposition." In Robotics and Automation, 2011International Conference on IEEE, 2011, pp. 1-4.

[38] Khatib, Oussama. "Real-time obstacle avoidance for manipulators and mobile robots." The international journal of robotics research. 1986, vol. 5.1.pp. 90-98.

[39] Chen YB, Luo GC, Mei YS, Yu JQ, Su XL. "UAV path planning using artificial potential field method updated by optimal control theory." International Journal of Systems Science. 2016,vol.47(6):1407-20.

[40] Raja R, Dutta A, Venkatesh KS. "New potential field method for rough terrain path planning using genetic algorithm for a 6-wheel rover." Robotics and Autonomous Systems. 2015 Oct 1;72:295-306.

[41] Louchene, A., Bouguechal, N.E., Dahmani, A., Yahiaoui, S. and Merrouchi, S., "Automated guided vehicle path planning without obstacles expansion." In Control Applications, Proceedings of the 1998 IEEE International Conference.1998; Vol. 2.pp. 1333-1337.

[42] Hoang, V.D., Hernandez, D.C., Hariyono, J. and Jo, K.H.”Global path planning for unmanned ground vehicle based on road map images." In Human System Interactions (HSI), 2014 7th International Conference. IEEE. 2014;pp. 82-87.

[43] Hermann M, Pentek T, Otto B. "Design principles for industrie 4.0 scenarios." InSystem Sciences (HICSS), 2016 49th Hawaii International Conference. IEEE. 2016; pp. 3928-3937.

[44] Sarvari PA, Ustundag A, Cevikcan E, Kaya I, Cebi S. "Technology Roadmap for Industry 4.0."Managing The Digital Transformation." Springer, Cham. 2018 ;pp. 95-103.

[45] Debnath, S.K., Omar, R. and Latip, N.B.A., "A Review on Energy Efficient Path Planning Algorithms for Unmanned Air Vehicles." In Computational Science and Technology Springer, Singapore. 2019; pp. 523-532.

[46] Latip, N.B.A., Omar, R. and Debnath, S.K.,. "Optimal Path Planning using Equilateral Spaces Oriented Visibility Graph Method.” International Journal of Electrical and Computer Engineering (IJECE), 7(6), pp.3046-3051,2017.

\section{BIOGRAPHIES OF AUTHORS}

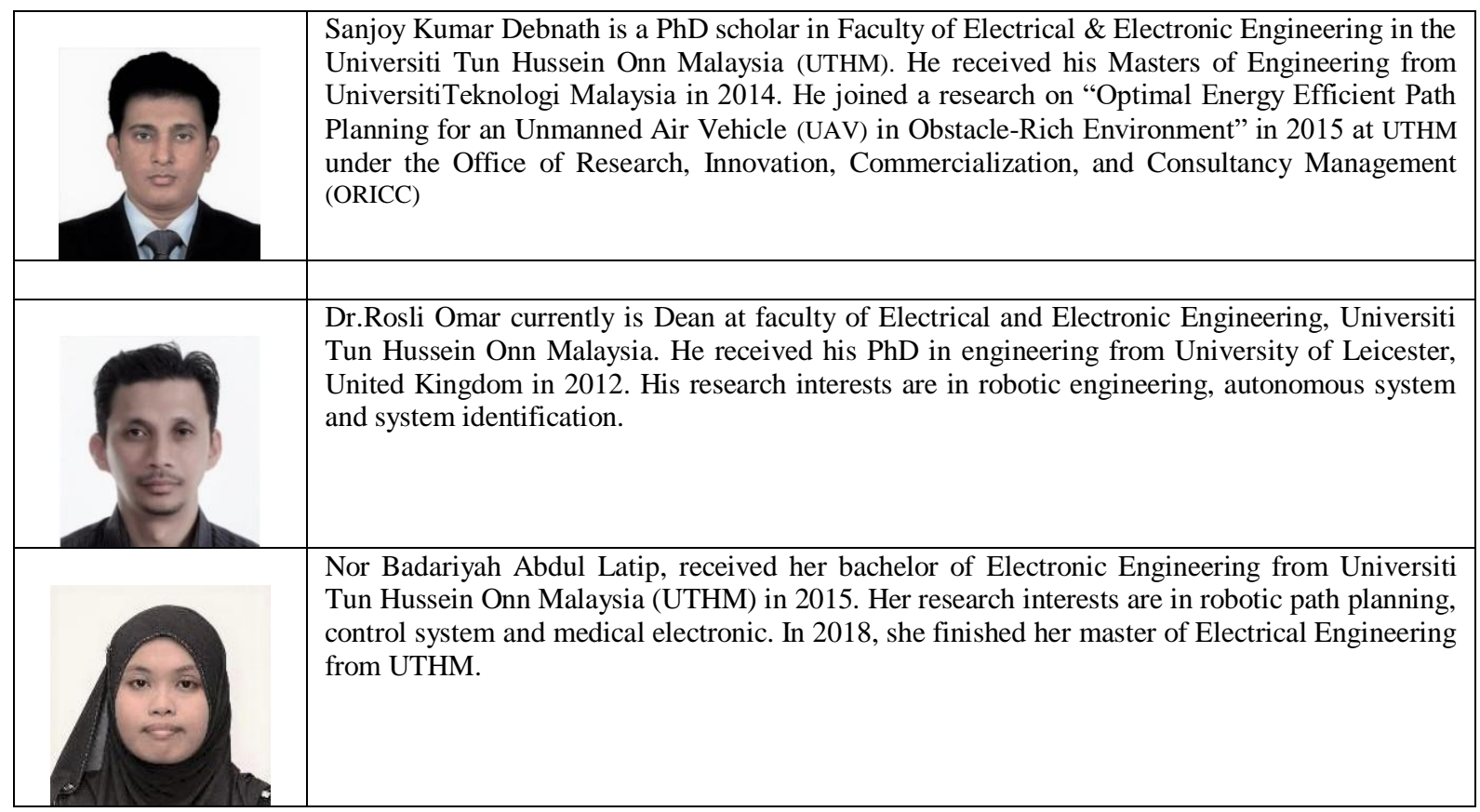

